\title{
Immunization against Intestinal Bacterial Endotoxin Prevents Alcoholic Fatty Liver in Rats
}

\author{
Gendo NISHIMURA $^{1)}$, Keiko NAKAHARA ${ }^{1)}$, Naoaki MISAWA ${ }^{2)}$, Miki MURANAKA ${ }^{3)}$, Kazuyuki UCHIDA ${ }^{3)}$, \\ Haruto KURODA ${ }^{1)}$ and Noboru MURAKAMI ${ }^{1) *}$ \\ ${ }^{1)}$ Departments of Veterinary Physiology, ${ }^{2)}$ Veterinary Public Health and ${ }^{3)}$ Veterinary Pathology, Faculty of Agriculture, University of \\ Miyazaki, Miyazaki 889-2155, Japan
}

(Received 29 August 2000/Accepted 27 November 2000)

ABSTRACT. Accumulating evidences indicate that an endotoxin originating from intestinal gram-negative bacteria may be involved in alcohol-induced liver injury including fatty liver. Therefore, whether immunization against intestinal bacterial endotoxin blocked fatty liver induced by chronic alcohol and diet including much-unsaturated fatty acid was investigated in rats. The titer of antibody against the endotoxin increased significantly after 13 weeks of continuous immunization. Daily alcohol treatment was initiated at 12 weeks and continued for 4 weeks. Plasma glutamic pyruvic transaminase (GPT), glutamic oxaloacetic transaminase (GOT) and triglyceride (TG) levels increased significantly in non-immunized rats receiving alcohol, but not in immunized rats. Continuous alcohol treatment gradually decreased the survival rate to $60 \%$ from 13 days after beginning administration in non-immunized, but not immunized, rats. A histochemical study revealed that continuous treatment with alcohol and unsaturated fatty acids caused fatty liver in non-immunized, but not immunized, rats. This study strongly supports the hypothesis that alcohol-induced fatty liver is due to a circulating endotoxin, and suggests that immunization for endotoxin prevent the alcoholic fatty liver.

KEY WORDS: alcohol, endotoxin, fatty liver.

J. Vet. Med. Sci. 63(3): 275-280, 2001

Although the animal models of alcohol-induced liver injury has been developed [26], the mechanisms has not been completely cleared yet, because of it's complexity [6, 10]. It is likely that females are more sensitive to alcohol than males $[15,27]$. Over the past decade, clinical studies have shown that endotoxin levels are increased in alcoholic patients [25]. Recently, it has been hypothesized that circulating endotoxins, originating from intestinal gram-negative bacteria and released by alcohol exposure, might induce liver injury $[2,5,25,27]$. Several lines of evidence to support this theory have now accumulated, as follows: 1) intestinal levels of lipopolysaccharides (LPS), which are components of bacterial cell walls, are increased by alcohol treatment $[5,19], 2)$ alcohol enhances the permeability of the intestinal mucous membrane to endotoxin [3, 4], 3) endotoxin activates Kupffer cells [1,9], 4) several antibiotics prevent alcohol-induced liver injury, as does intestinal sterilization [2], 5) prevention of the growth of intestinal gram-negative bacteria by treatment with Lactobacillus inhibits alcohol-induced liver disease [20], 6) selective inactivation of macrophages (the major population of which is Kupffer cells) or blockade of CD14 or tumor necrosis factor (TNF- $\alpha$ ) by several agents or antibody prevents alcoholinduced liver injury [1, 4, 28], 7) after Kupffer cell activation, various chemical mediators, such as interleukins, prostaglandin $\mathrm{E}_{2}$, TNF- $\alpha, \mathrm{Ca}^{2+}$, free radical and so on, are released and have a toxic effect on the liver $[1,7,12,14,16$, $17,18,22]$. These observations indicate that there may be a

\footnotetext{
* Correspondence to: Murakami, N., Department of Veterinary Physiology, Faculty of Agriculture, University of Miyazaki, Miyazaki 889-2155, Japan.
}

cascade mechanism involved in alcohol-induced liver injury, that is: alcohol $\rightarrow$ endotoxin $\rightarrow$ Kupffer cell sensitization $\rightarrow$ toxic mediator activity $\rightarrow$ liver injury [25]. However, there was no direct evidence in vivo to explain this hypothesis. In the present study, we made immunized rats against an endotoxin prepared from intestinal bacteria and supplied diet including much-unsaturated fatty acid in rats, and investigated whether continuous neutralization of this circulating endotoxin prevented fatty liver $[8,11]$.

\section{MATERIALS AND METHODS}

Fifty-day-old female Wistar rats were maintained under a $12 \mathrm{hr}$ light: $12 \mathrm{hr}$ dark cycle (lights on at 07:00 hr) at $22 \pm$ $1^{\circ} \mathrm{C}$. Food (CE-2, Nihonkurea Co., Japan) and water were provided ad libitum throughout the experiment. However, after initiating alcohol treatment, a separate fluid meal was also given (details described below).

Immunization: The endotoxin antigen for immunization was prepared from rat intestinal bacteria, since specific endotoxin concerning to alcoholic liver injury has not been identified yet. Briefly, intestinal bacteria were plated in DHL agarose wells and Bacteroides agarose wells (Nissui. Pharmacol. Co., Tokyo) and cultured at $37^{\circ} \mathrm{C}$ using aerobic and anaerobic culture methods, respectively. The proliferating bacteria were collected into a $50-\mathrm{m} l$ centrifuge tube containing $40 \mathrm{~m} l$ saline and centrifuged at $1,900 \times \mathrm{g}$ for $5 \mathrm{~min}$. After removing the supernatant, the sedimented bacteria were washed twice with saline. The bacterial concentration was adjusted with saline in a spectrophotometer (Hitachi Co., Tokyo), using an absorbance of $600 \mathrm{~nm}$. The bacteria were then homogenized and their cytosolic protein was 


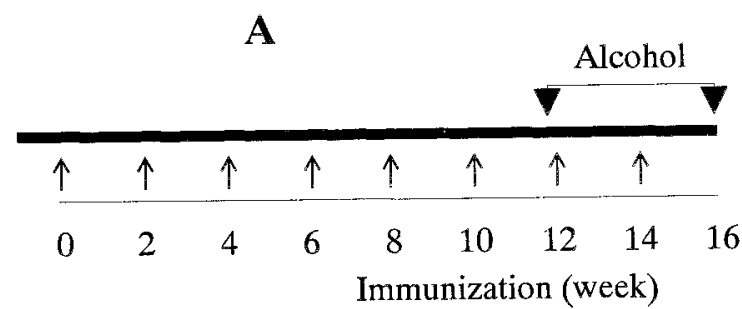

$(\mathrm{g} / \mathrm{Kg})$


Fig. 1. Schedules for immunization (A), alcohol treatment (B) and measurement of antibody titer (C). The endotoxin (or saline)incomplete Freund's adjuvant complex was injected every two weeks (as indicated by the arrows) for 16 weeks (A). Daily alcohol administration was started 12 weeks after beginning immunization and continued for 4 weeks, increasing the dose gradually to avoid shock and loss of body weight (B). The antibody titer was checked once a week, but only data from 12 weeks onwards are shown $(\mathrm{C})$. A triangle connected with dotted line: titer of endotoxin-immunized rats receiving saline, a diamond connected with straight line: titer of endotoxin-immunized rats receiving alcohol, white and black circle connected straight line: non-immunized rats receiving alcohol and saline, respectively. Asterisks indicate the significant difference $(P<0.05$ vs non immunized rat).

denatured at $121^{\circ} \mathrm{C}$ for $15 \mathrm{~min}$ in an autoclave to obtain a crude cell wall endotoxin solution. The crude endotoxin solution was conjugated with incomplete Freund's adjuvant (Difco Lab., Michigan, U.S.A) at the ratio of 1:1:2 (endotoxin derived from aerobic bacteria: endotoxin derived from anaerobic bacteria: incomplete Freund's adjuvant). The mixed antigen-adjuvant was injected into the foot pad of 48 rats every 2 weeks $(0.15 \mathrm{~m} /$ injection $)$ following the schedule shown in Fig. 1A. As a control, a mixed solution of saline-incomplete Freund's adjuvant was injected into 48 further rats at the same times.

Treatment with alcohol and a highr-calorie diet: Twelve weeks after initiating immunization, direct administration of alcohol into the stomach through a tube was begun. Saline was used instead of alcohol in the controls. As body weight and food intake were decreased by direct administration of alcohol into the stomach during a preliminary experiment, glucose ( $1 \mathrm{~g} /$ daily dose) was dissolved in the alcohol or saline solution. The alcohol concentration was gradually increased according to the schedule shown in Fig. 1B, starting with a very low dose, especially in the first week. In addition, the total daily dose was divided into two or three portions per day, to avoid alcoholic shock or a decrease in body weight $[16,22]$. Using a three-way stopcock attached to the gastric tube, $2.5 \mathrm{ml}$ alcohol or saline containing $1 \mathrm{~g}$ glucose was administered from one syringe, followed immediately by $1 \mathrm{~m} l$ corn oil (containing 50\% linoleic acid) and ZEVAS Sport supplement Protein (Meiji Co., Tokyo Japan) including vitamins, minerals, protein and carbohydrates from a separate syringe $[8,11]$. The total calorie was approximately $38.4 \mathrm{~kJ} / \mathrm{kg}$ body weight/day.

Measurement of the serum anti-LPS titer: Blood $(60 \mu l)$ was collected once a week from all rats, using the tail tip incision method without anesthesia. The blood was collected in hematocrit tubes and the plasma was separated immediately. A passive hemagglutination technique was used for titration of antisera [15]. Heart-extracted antigens were incubated for $1 \mathrm{hr}$ at $37^{\circ} \mathrm{C}$ with an equal volume of a $1 \%$ suspension of sheep erythrocytes previously washed in saline. The sensitized erythrocytes were washed and resuspended in saline to a final volume equivalent to a $0.5 \%$ erythrocytes suspension. Two-fold serum dilution was performed in a 96 well microtitration plate and then sensitized erythrocytes were added to each well of diluted antisera. The highest dilution of antiserum showing agglutination was taken as the titer.

Biochemical and histochemical analysis: All rats were killed by decapitation on day 29 after starting the alcohol/ saline, glucose and corn oil treatment, and blood and liver samples were collected. Plasma glutamic oxaloacetic transaminase (GOT), glutamic pyruvic transaminase (GPT), creatinine phosphokinase (CPK) and triglyceride (TG) levels were measured using commercial assay kits (Fuji-DRICHEM5500V and DRI-CHEM SLIDE; Fuji Film Co., Tokyo) in the veterinary hospital at our university. Blood alcohol levels were determined on a clinical laboratory of Miyazaki Medical College. Interleukin (IL)- 8 and TNF- $\alpha$ were determined using enzyme immunoassay kits (Wako Pharmacol. Co. and Yanaihara Res. Center, respectively, Japan). Tissue samples from each organ were fixed with $4 \%$ formalin and routinely processed into paraffin sections, which were then stained with hematoxylin and eosin. Some liver tissue sections were stored at $-80^{\circ} \mathrm{C}$, and these frozen sections were stained with Sudan Black to detect their lipid 


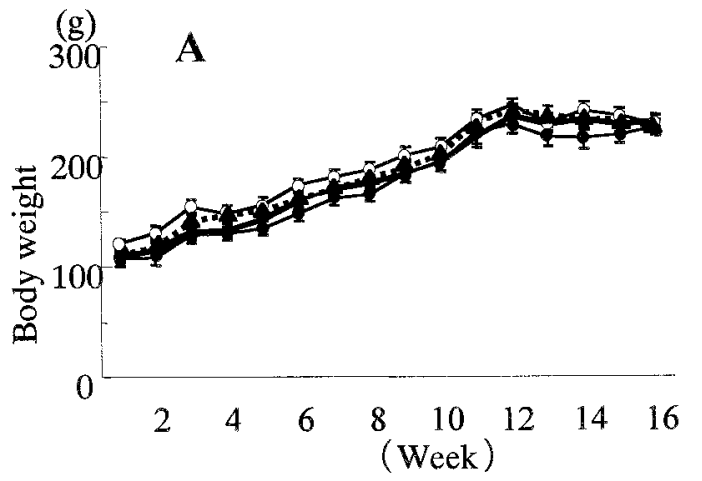

Saline+non-immun. or
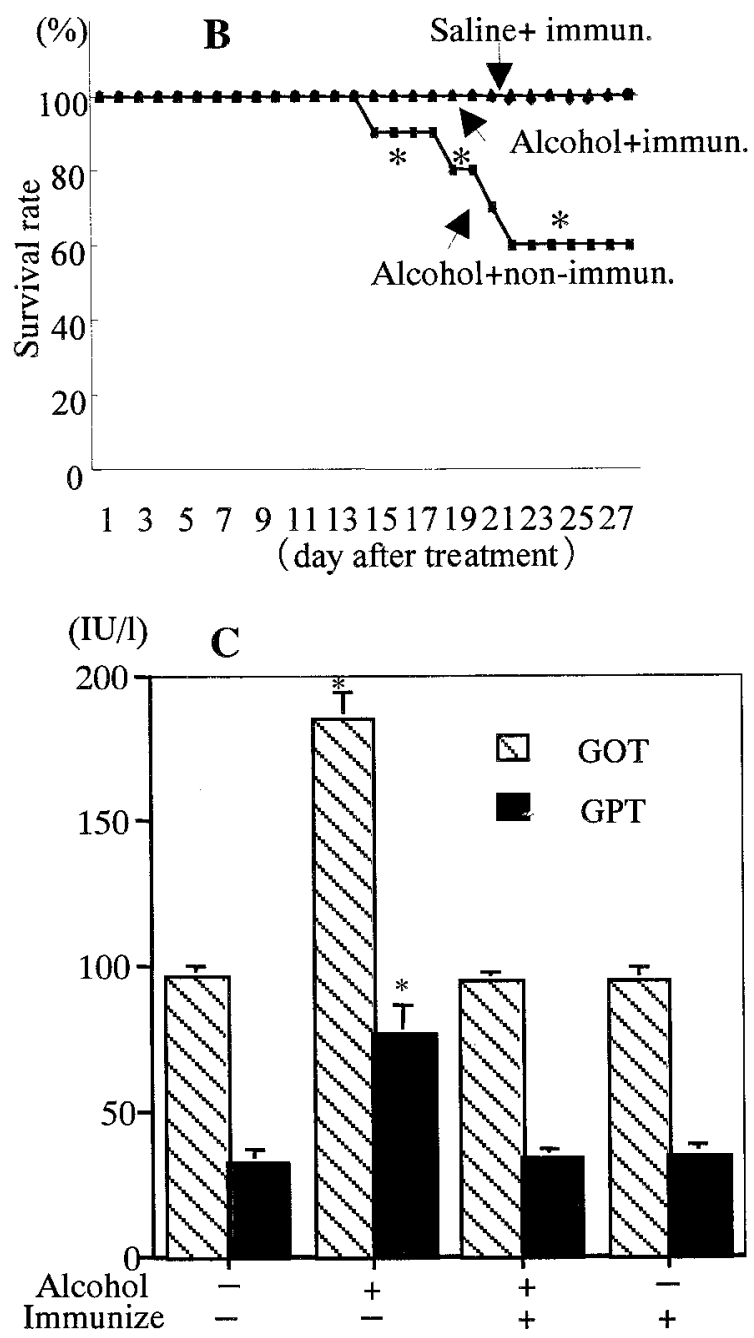

Fig. 2. Effect of alcohol treatment on body weight (A), the survival rate (B) and GOT and GPT levels (C) in immunized and nonimmunized rats. There were no significant differences in body weight after alcohol treatment. The black circles and squares represent immunized rats receiving alcohol and saline, respectively. The white circles and squares represent non-immunized (saline-treated) rats receiving alcohol and saline, respectively (A). After four weeks of alcohol treatment, GOT and GPT were content. The degree of fatty liver was evaluated 4 grade by the rate of appearance of lipid drops in the hepatic lobules (less than $25 \%=$ grade $1,25 \sim 50 \%=$ grade $2,50 \sim 75 \%=$ grade 3 , over $75 \%=$ grade 4 ). Hepatic lobules with a lipid drop content of over $50 \%$ were classed as fatty liver.

The data were expressed as the mean \pm SEM. The statistical analysis was carried out by ANOVA with the post hoc Fisher test.

\section{RESULTS}

Continuous treatment with an endotoxin antigen prepared from intestinal bacteria, conjugated with incomplete Freund's adjuvant, increased the titer of antibodies against the endotoxin in rats. A significant increase in the antibody titer was observed 13 weeks after starting immunization, and the titer continued to increase thereafter (Fig. 1C). However, the titer was lower in the group of endotoxin-immunized rats receiving alcohol than in those receiving no alcohol (Fig. 1C). In general, although chronic alcohol treatment causes loss of body weight in rats (preliminary study), administration of glucose and corn oil including high-unsaturated fatty acid together with the alcohol prevented this weight loss (Fig. 2A). However, regardless of the prevention of weight loss, the survival rate was decreased to about $60 \%$ ( 10 of the 24 rats died between 3 and 4 weeks after starting alcohol treatment) among the non-immunization rats. This death started from the day of maximum alcohol treatment. On the other hand, no deaths were observed throughout the experiment among endotoxin-immunized rats receiving alcohol (Fig. 2B). The biochemical examination clearly showed that plasma GOT, GPT and TG levels were increased about two- or three-fold in non-immunization rats receiving alcohol, compared with endotoxin-immunized rats receiving alcohol or saline, and non-immunization rats receiving saline (Fig. 2C; Fig. 3A). The blood alcohol levels were $248 \pm 86$ and $259 \pm 48 \mathrm{mg} / 100 \mathrm{ml}$ on day 9 , and $396 \pm 41$ and $232 \pm 64 \mathrm{mg} / 100 \mathrm{ml}$ on day 23 $(\mathrm{P}<0.05)$ in non-immunization and immunized rats, respectively. There were no significant differences in CPK levels between any of the four groups (Fig. 3A). Although TNF- $\alpha$ and IL8 were measured four weeks after beginning alcohol treatment as a indicator of macrophage activation and stage of liver injury, their levels increased not only in the alcoholtreated rats but also in the immunized rats. Indeed, the only group with no increase were non-immunization rats receiving saline (Fig. 3B). The histochemical study revealed that treatment with alcohol for four weeks induced the appearance of many lipid drops in hepatic lobules in 13 out of the 14 surviving non-immunization rats (10 of the 24 rats died before sacrifice) (Fig. 4C, Fig. 3C). The remaining one rat showed moderate lipid changes (data not shown). On the other hand, 9 out of the 24 immunized rats receiving alcohol

increased while the survival rate was decreased in non-immunized rats $(P<0.005$ vs the other groups), but not in the immunized rats $(\mathrm{B}, \mathrm{C})$. 

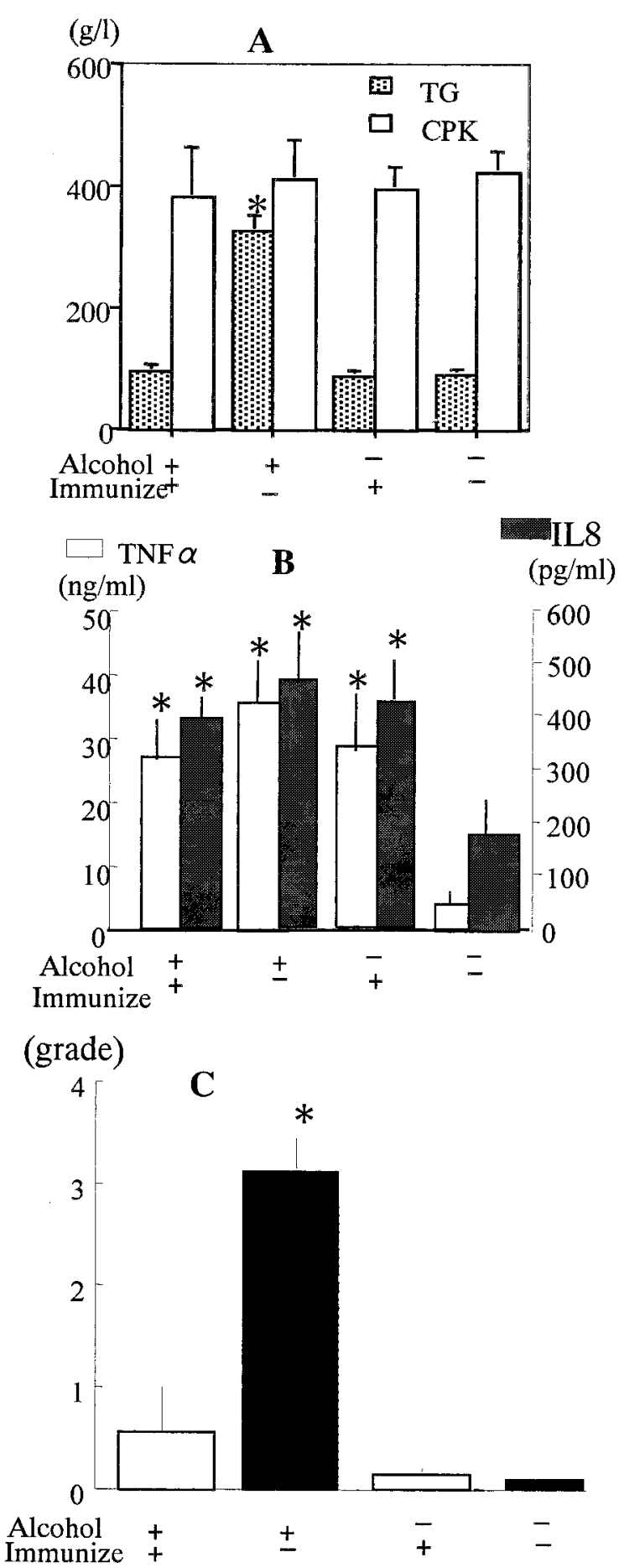

Fig. 3. Effect of alcohol treatment on TG and CPK levels (A), TNF- $\alpha$ and IL8 levels (B) and grade of fatty liver (C) in endotoxin-immunized and non-immunized rats. After four weeks of alcohol treatment, TG was increased in non-immunized rats $(P<0.005$ vs the other groups), but not in immunized rats (A). TNF $\alpha$ and IL8 levels increased significantly in both alcoholtreated and immunized rats (B). Fatty liver occurred in nonimmunized rats receiving alcohol. The points indicate the relative showed slight lipid changes in hepatic lobules, while the remaining 15 showed hardly any change (Fig. 4A). None of the 48 rats which received saline instead of alcohol showed any significant liver changes (Fig. 4 B, D).

\section{DISCUSSION}

Continuous treatment with an endotoxin antigen prepared from intestinal bacteria, conjugated with incomplete Freund's adjuvant, increased the titer of antibodies against the endotoxin in rats. However, the titer was lower in the group of endotoxin-immunized rats receiving alcohol than in those receiving no alcohol. This may be probably due to an alcohol-induced increase in circulating endotoxin levels, followed by antibody binding to the endotoxin. Therefore, the lower titer in alcohol-treated rats may be due to a decrease in the amount of free antibody. Although chronic alcohol treatment with glucose and corn oil including high-unsaturated fatty acid together with the alcohol prevented the weight loss, the survival rate was decreased to about $60 \%$ among the non-immunized rats. On the other hand, no deaths were observed throughout the experiment among endotoxin-immunized rats receiving alcohol. The reason for this death may not be due to liver injury by judging from GOT levels and degree of fatty liver [4]. After the pathological dissection, we considered that the cause of death might be due to complex of vascular change in many tissues by endotoxin and brain damage by alcohol. The biochemical examination clearly showed that plasma GOT, GPT and TG levels were increased about two- or three-fold in non-immunized rats receiving alcohol compared with endotoxinimmunized rats receiving alcohol or saline, and non-immunized rats receiving saline. There were no significant differences in CPK levels between any of the four groups. These results suggest that alcohol caused some liver injury in nonimmunized rats, while endotoxin immunization prevented alcohol-induced liver injury.

Although TNF- $\alpha$ and IL8 were measured four weeks after beginning alcohol treatment as a indicator of macrophage activation [23] and stage of liver injury [13], their levels increased not only in the alcohol-treated rats but also in the immunized rats. Indeed, the only group with no increase were non-immunized rats receiving saline. This result was unexpected, but it is possible that the origin of the increase in TNF- $\alpha$ and IL8 may differ between alcohol-treated nonimmunized rats and endotoxin-immunized rats. In the former, the increase may have originated from macrophages among the Kupffer cells of the liver, while in the latter, it may have arisen due to an inflammatory reaction caused by injection of the Freund's adjuvant-endotoxin conjugate into the foot-pad $[13,17,23]$.

The histochemical study revealed that treatment with alcohol for four weeks induced the appearance of many lipid

scores for the presence of lipid drops in the hepatic lobule (C). Asterisks indicate the significant difference $(P<0.05$ vs others). 
A

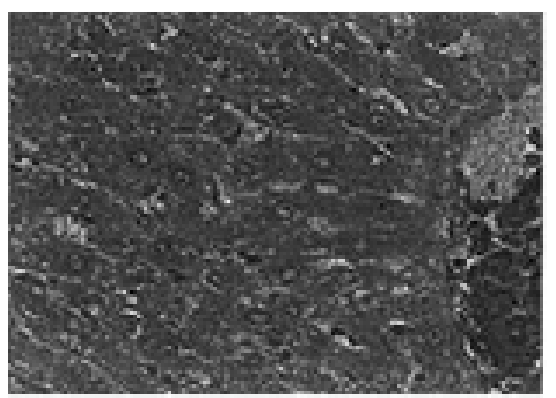

B

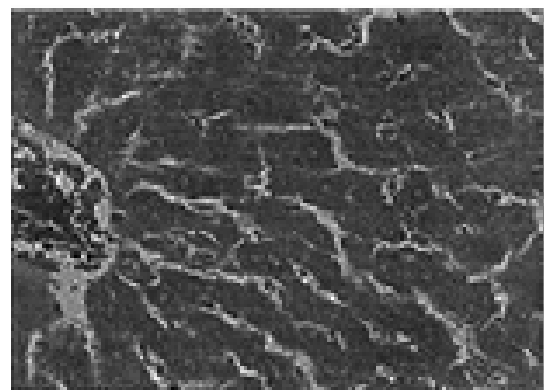

$\mathrm{C}$

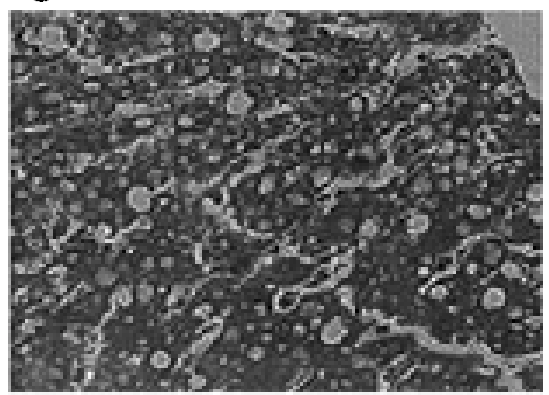

$\mathrm{D}$

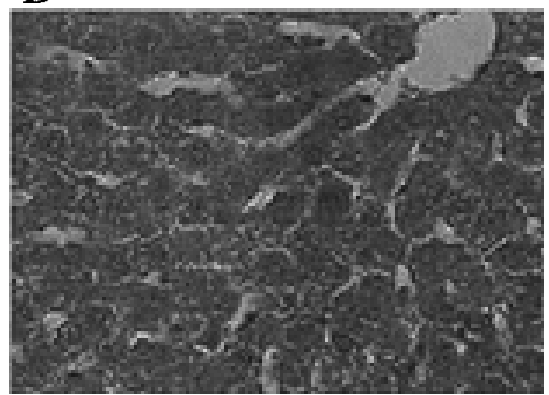

Fig. 4. Liver histology after administration of alcohol or saline, together with glucose and corn oil, in immunized and non-immunized rats. A: endotoxin-immunized rats receiving alcohol, B: immunized rats receiving saline, $\mathrm{C}$ : non-immunized rats receiving alcohol, D: non-immunized rats receiving saline. Large numbers of lipid drops can be observed in $\mathrm{C}$ (Final magnification $\times 300)$.

drops in hepatic lobules in 13 out of the 14 surviving nonimmunized rats. The remaining rat showed moderate lipid changes. On the other hand, 9 out of the 24 immunized rats receiving alcohol showed slight lipid changes in hepatic lobules, while the remaining 15 showed hardly any change. None of the 48 rats that received saline instead of alcohol showed any significant liver changes. These histological results strongly suggest that endotoxin immunization prevent alcohol-induced fatty liver. Furthermore, as no fatty liver was observed in the non-immunized rats receiving saline together with glucose and corn oil, fatty liver appears to be predominantly due to alcohol. The mechanisms underlying liver damage after acute alcoholic exposure also differ from those that occur during chronic exposure to alcohol $[8$, $9,22,29]$. It has been suggested that, in order to induce alcoholic fatty liver, a particular nutritional background, such as supplementation with unsaturated fatty acids, is necessary $[8,11,25]$. Although an increase in circulating LPS stimulates the production of toxic mediators, including TNF- $\alpha[17,23]$, and thereafter causes endotoxemia, increases in LPS are usually prevented by many protective bodily mechanisms, such as inflammatory responses involving macrophages, opsonic reactions involving complement, neutrophil-induced bacterial aggregation, and so on [24, 25]. However, during chronic alcohol administration, large amounts of endotoxins are carried from the intestine to the liver via the portal blood flow [25]. Under these conditions,
Kupffer cells might become a direct target for endotoxins. The present study provides direct evidence confirming the hypothesis that fatty liver following chronic alcohol exposure and high calorie diet may be due to continuous activation of the Kupffer cells by an alcohol-induced endotoxin originating from intestinal bacteria $[2,5,25,27]$. In this study, we could not determine blood endotoxin levels after immunization, since blood has already included antibody for endotoxin. Further studies are required to elucidate the further mechanism of alcohol induced fatty liver.

\section{REFERENCES}

1. Adachi, Y., Bradford, B. U., Gao, W., Bojes, H. K. and Thurman, R. G. 1994. Inactivation of Kupffer cells prevents early alcohol-induced liver injury. Hepatology 20: 453-460.

2. Adachi, Y., Moore, L. E., Bradford, B. U., Gao, W. and Thurman, R. G. 1995. Antibiotics prevent liver injury in rats following long-term exposure to ethanol. Gastroenterology 108: 218 224.

3. Arai, M. 1986. Effect of ethanol on the intestinal uptake of endotoxin (Abstract). Nippon Shokakibyo Gakkai Zasshi 83: 1060 .

4. Bjarnason, I., Ward, K. and Peters, T. J. 1984. The leaky gut of alcoholism: possible route of entry for toxic compounds. Lancet 1: 179-182.

5. Bode, C. H., Kugler, V. and Bode, J. C. 1987. Endotoxemia in patients with alcoholic and non-alcoholic cirrhosis and in subjects with no evidence of chronic liver disease following acute 
alcohol excess. J. Hepatol. 4: 8-14.

6. Crabb, D.W. 1999. Pathogenesis of alcoholic liver disease: new mechanisms of injury. Keio J. Med. 48: 184-188.

7. Decker, T., Lohmann-Matthes, M. L., Karck, U., Peters, T. and Decker, K. 1989. Comparative study of cytotoxicity, tumor necrosis factor, and prostaglandin release after stimulation of rat Kupffer cells, murine Kupffer cells, and murine inflammatory liver macrophages. J. Leukoc. Biol. 45: 139-146.

8. Earnest, D. L., Abril, E. R., Jolley, C. S. and Martinez, F. 1993. Ethanol and diet-induced alterations in Kupffer cell function. Alcohol Alcohol. 28: 73-83.

9. Enomoto, N., Ikejima, K., Bradford, B. U., Rivera, C. A., Kono, H., Brenner, D. A. and Thurmann, R. G. 1998. Alcohol causes both tolerance and sensitization of rat Kupffer cells via mechanisms dependent on endotoxin. Gastoroenterology 115: 443-451.

10. Enomoto, N., Ikejima, K., Bradford, B. U., Rivera, C. A., Kono, H., Goto, M., Yamashina, S., Schmmer, P., Kitamura, T., Oide, H., Takei, Y., Hirose, M., Shimizu, H., Miyazaki, A., Brenner, D. A., Sato N. and Thurmann, R. G. 2000. Role of Kupffer cells and gut-derived endotoxins in alcoholic liver injury. J. Gastoroenterol. Hepatol. (Suppl.) D20-25.

11. French, S. W. 1993. Nutrition in the pathogenesis of alcoholic liver disease. Alcohol Alcohol. 28: 97-109.

12. Hill, D. B., Marsano, L., Cohen, D., Allen, J., Shedlofsky, S. and McClain, C. J. 1992. Inceased plasma interleukin-6 concentrations in alcoholic hepatitis. J. Lab. Clin. Med. 119: 547552.

13. Huang, Y. S., Chan, C. Y., Wu. J. C., Pai, C. H., Chao, Y. and Lee, S. D. 1996. Serum levels of interleukin-8 in alcoholic liver disease: relationship with disease stage, biochemical parameters and survival. J. Hepatol. 24: 377-384

14. Iimuro, Y., Ikejima, K., Rose, M. L., Bradford, B. U. and Thurmann, R. G. 1996. Nimodipine, a dihydropyridine-type calcium channel blocker, prevents alcoholic hepatitis due to chronic intragastric ethanol exposure in the rat. Hepatology 24: 391-397.

15. Iimuro, Y., Frankenberg, M.V., Arteel, G. E., Bradford, B. U., Wall, C. A. and Thurman, R. G. 1997. Female rats exhibit greater susceptibility to early alcohol-induced injury than males. Am. J. Physiol. 272: G1186-G1194.

16. Knecht, K. T., Bradford, B. U., Mason, R. P. and Thurman, R. G. 1990. In vivo formation of a free radical metabolite of ethanol. Mol. Pharmacol. 38: 26-30.

17. Martinez, F., Abril, E. R., Earnest, D. L. and Watson, R. R. 1992. Ethanol and cytokine secretion. Alcohol 9: 455-458.

18. McClain, C. J., Cohen, D. A., Dinarello, C. A., Cannon, J. G.,
Shedlofsky, S. I. and Kaplan, A. M. 1986. Serum interleukin1(IL-1) activity in alcoholic hepatitis. Life Sci. 39: 1479-1485.

19. Nanji, A. A., Khettry, U., Sadrzadeh, S. M. and Yamanaka, T. 1993. Severity of liver injury in experimental alcoholic liver disease. Correlation with plasma endotoxin, prostaglandin E2, leukotriene B4, and Thromboxane B2. Am. J. Pathol. 142: 367-373.

20. Nanji, A. A., Khettry, U. and Sadrzadeh, S. M. H. 1994. Lactobacillus feeding reduces endotoxemia and severity of experimental alcoholic liver disease. Proc. Soc. Exp. Biol. Med. 205: 243-247.

21. Naveau, S., Giraud, V., Borotto, E., Aubert, A., Capron, F. and Chaput, J. C. 1997. Excess weight risk factor for alcoholic liver disease. Hepatology 25: 108-111.

22. Nelson, S., Bagby, G. J., Bainton, B. G. and Summer, W. R. 1989. The effect of acute and chronic alcoholism on tumor necrosis factor and inflammatory response. J. Infect. Dis. 160: 422-429.

23. Stahnke, L. L., Hill, D. B. and Allen, J. I. 1991. TNFalpha and IL-6 in alcoholic liver disease. pp. 472-475. In: Cells of the Hepatic Sinusoid (Wisse, E.. Knook, D. L. and McCusky. R. S. eds), Leiden, The Netherlands: Kupffer Cell Foundation.

24. Tabakoff, B., Hoffman, P. L., Lee, J. M., Saito, T., Willard, B. and Leon-Jones, F. D. 1988. Differences in platelet enzyme activity between alcoholics and nonalcoholics. New Engl. J. Med. 318: 134-139.

25. Thurman, R. G. 1998. Mechanisms of hepatic toxicity II. Alcoholic liver injury involves activation of Kupffer cells by endotoxin. Am. J. Physiol. 275: G605-G611.

26. Tsukamoto, H., Reiderberger, R. D., French, S. W. and Largman, C. 1984. Long-term cannulation model for blood sampling and intragastric infusion in the rat. Am. J. Physiol. 247: R595-R599.

27. Turmann, R. G., Bradford, B. U., Iimuro, Y., Knecht, K. T., Conner, H. D., Adachi, Y., Wall, C., Arteel, G. E., Raleigh, J. A., Forman, D. T. and Mason, R. P. 1997. Role of Kupffer cells, endotoxin and free radicals in hepatotoxicity due to prolonged alcohol consumption: Studies in female and male rats. J. Nutr. 127: 903S-906S.

28. Wang, P., Kitchens, R. L. and Munford, R. S. 1998. Phosphatidylinositides bind to plasma membrane CD14 and can prevent monocyte activation by bacterial lipopolysaccharide. J. Biol. Chem. 273: 24309-24313.

29. Yamada, S., Mochida, S., Ohno, A., Hirata, K., Ogata, I., Ohta, Y. and Fujiwara, K. 1991. Evidence for enhanced secretory function of hepatic macrophages ager long-term ethanol feeding in rats. Liver 11: 220-224. 